H. Kitahara, H. Kawakami and J. S. Pak

Nagoya Math. J.

Vol. 113 (1989), 7-13

\title{
ON A CONSTRUCTION OF COMPLETE SIMPLY-CONNECTED RIEMANNIAN MANIFOLDS WITH NEGATIVE CURVATURE
}

\author{
HARUO KITAHARA, HAJIME KAWAKAMI AND JIN SUK PAK
}

Let $M$ be a complete simply-connected riemannian manifold of even dimension $m$. J. Dodziuk and I.M. Singer ([D1]) have conjectured that $H_{2}^{p}(M)=0$ if $p \neq m / 2$ and $\operatorname{dim} H_{2}^{m / 2}(M)=\infty$, where $H_{2}^{*}(M)$ is the space of $L_{2}$-harmonic forms on $M$.

Recently, M. T. Anderson ([An]) constructed manifolds which are counterexamples to the J. Dodziuk-I. M. Singer conjecture. In this paper, we will discuss how to construct complete simply-connected riemannian manifolds with negative sectional curvature, by the idea of M. T. Anderson and a private advice of J. Dodziuk ([D2]).

THEOREM. Let $B$ be a complele riemannian $C^{\infty}$-manifold with $C^{\infty}$-connected boundary $\partial B$ and $f a C^{\infty}$-function on $B$. Suppose that $B$ and $f$ satisfy the following conditions;

(B.1) $B$ has the riemannian simple double $2 B$, that is the canonically endowed continuous metric of $2 B$ is smooth.

(B.2) The sectional curvature $K_{B}$ of $B$ is negative, or $B:=[0, \infty)$,

(B.3) $B$ is simply-connected,

(F.1) $f$ is a function of the geodesic distance $r$ from $\partial B$,

(F.2) $f$ is an odd function of $r$ on a neighborhood of $r=0$ and satisfies that $f^{\prime}(0)=1, f^{\prime \prime}(r)>0$ for $r>0$, and $f^{\prime \prime \prime}(0)>0$.

Let $M:=(B \backslash \partial B) \times_{f|B| \partial B} S^{n}(1)$. Then there is the unique complete simplyconnected riemannian manifold $\mathscr{M}$ with negative curvature which is the completion of $M$.

Remark. Any function on $[0, \infty)$ can be considered as a function satisfying (F.1) under the assumptions (B.1)-(B.3).

Manifolds are supposed to be connected paracompact Hausdorff spaces.

Received January 19, 1987. 
1.

J. Kazdan-F. Warner ([K-W]) proved that, for a $C^{\infty}$-metric $g$ on $R^{2} \backslash\{0\}$, there is a $C^{\infty}$-metric $\tilde{g}$ on $R^{2}$ such that $\tilde{g}$ restricted to $R^{2} \backslash\{0\}$ is g. First, we will generalize their result.

Lemma 1.1 (cf. [K-W], [O-N, p. 31]). If $f(t)$ is a real valued $C^{\infty}$-even function on $R$, then $f(r)$ is a $C^{\infty}$-function on $R^{n}$, where $r:=\left(\left(x^{1}\right)^{2}+\cdots+\right.$ $\left.\left(x^{n}\right)^{2}\right)^{1 / 2}$.

Lemma 1.2. Let $f: R^{m} \times R^{1+n} \rightarrow R$ be a continuous function. If $f$ satisfies the following conditions;

(1.2.1) $f$ is of class $C^{\infty}$ on $\left(R^{m} \times R^{1+n}\right) \backslash\left(R^{m} \times\{0\}\right)$,

(1.2.2) $f$ is invariant under $\left\{I_{m}\right\} \times O(n+1)$, where $\left\{I_{m}\right\}$ is the unit group on $R^{m}$ and $O(n+1)$ is the rotation group on $R^{1+n}$,

(1.2.3) $f$ is of class $C^{\infty}$ on $R^{m} \times l$ for any straight line $l \subset R^{1+n}$ through the origin, then $f$ is of class $C^{\infty}$ on $R^{m} \times R^{1+n}$.

Proof. We introduce two coordinates on $R^{m} \times R^{1+n}$, one is the usual Cartesian coordinates $\left(x^{1}, \cdots, x^{m}, z^{0}, z^{1}, \cdots, z^{n}\right)$ and one is $\left(x^{1}, \cdots, x^{m}, r\right.$, $\left.y^{1}, \cdots, y^{n}\right)$ where $\left(r, y^{1}, \cdots, y^{n}\right),(r>0)$, the polar coordinates on $R^{1+n}$. By (1.2.2), we can consider that $f$ is a function with only $\left(x^{1}, \cdots, x^{m}, r\right)$ variables.

Step 1. We take a point $x_{o}:=\left(x_{o}^{1}, \cdots, x_{o}^{m}\right)$ and fix it. (1.2.3), (1.2.2) and Lemma 1.1 imply that $f_{o}(r):=f\left(x_{o}, r\right), r:=\left(\left(z^{1}\right)^{2}+\cdots+\left(z^{n}\right)^{2}\right)^{1 / 2}$, can be considered to be of class $C^{\infty}$ on $R^{1+n}$. Since $\left(\partial / \partial x^{i}\right) f$ are invariant under $\left\{I_{m}\right\} \times O(n+1)$ and are of class $C^{\infty}$ on $R^{m} \times l$ for a fixed $l$, if we choose any sequence $\left\{\left(x_{n}, z_{n}\right)\right\}$ in $R^{m} \times R^{1+n}$ converging to $\left(x_{o}, 0\right)$, then we have

$$
\begin{aligned}
&\left|\left(\frac{\partial}{\partial x^{j}}\right) f\left(x_{n}, z_{n}\right)-\left(\frac{\partial}{\partial x^{j}}\right) f\left(x_{o}, 0\right)\right| \\
&=\left|\left(\frac{\partial}{\partial x^{j}}\right) f\left(x_{n}, \pi\left(z_{n}\right)\right)-\left(\frac{\partial}{\partial x^{j}}\right) f\left(x_{o}, 0\right)\right| \longrightarrow 0 \\
&\left(\left(x_{n}, z_{n}\right) \longrightarrow\left(x_{o}, 0\right)\right),
\end{aligned}
$$

where $\pi: R^{1+n} \rightarrow l_{+}:=\{r \in l \mid r \geq 0\}$ is the canonical projection. Thus, together with by (1.2.1), we have that $\left(\partial / \partial x^{j}\right) f$ are continuous on $R^{m} \times R^{1+n}$, and, inductively, $\left(\partial^{\alpha_{1}+\cdots+\alpha_{k}} /\left(\partial x^{i_{1}}\right)^{\alpha_{1}} \cdots\left(\partial x^{i_{k}}\right)^{\alpha_{k}}\right) f$ are continuuos on $R^{m} \times R^{1+n}$. 
Step 2. We set

$$
F(x, r):=\left(\frac{\partial^{\alpha_{1}+\cdots+\alpha_{k}}}{\left(\partial x^{i_{1}}\right)^{\alpha_{1}} \cdots\left(\partial x^{i_{k}}\right)^{\alpha_{k}}}\right) f(x, r), \alpha_{1}+\cdots+\alpha_{k} \geq 0
$$

Note that $F_{o}(r)$ is of class $C^{\infty}$ on $R^{1+n}$. For example, since

$$
\frac{\partial^{2}}{\partial z^{\alpha} \partial z^{\beta}} F(x, r)= \begin{cases}\left(\frac{1}{r} \frac{\partial}{\partial r}\right)^{2} F(x, r) z^{\alpha} z^{\beta}, & \alpha \neq \beta, \\ \left(\frac{1}{r} \frac{\partial}{\partial r}\right)^{2} F(x, r)\left(z^{\alpha}\right)^{2}+\frac{1}{r} \frac{\partial}{\partial r} F(x, r), & \alpha=\beta,\end{cases}
$$

and $(1 / r \cdot \partial / \partial r)^{p} F(x, r)(p=0,1,2, \cdots)$ are even functions in $r$, by the same way as Step $1,\left(\partial^{2} / \partial z^{\alpha} \partial z^{\beta}\right) F(x, r)$ are continuous on $R^{m} \times R^{1+n}$. More generally, we have

$\left(\frac{\partial^{\beta_{1}+\cdots+\beta_{s}+\alpha_{1}+\cdots+\alpha_{k}}}{\left(\partial z^{j_{1}}\right)^{\beta_{1}} \cdots\left(\partial z^{j_{s}}\right)^{\beta_{s}}\left(\partial x^{i_{1}}\right)^{\alpha_{1}} \cdots\left(\partial x^{i_{k}}\right)^{\alpha_{k}}}\right) f \quad\left(\beta_{1}+\cdots+\beta_{s}>0, \alpha_{1}+\cdots+\alpha_{k} \geq 0\right)$

are continuous on $R^{m} \times R^{1+n}$. Therefore, $f$ is of class $C^{\infty}$ on $R^{m} \times R^{1+n}$.

Proposition 1.3. Let $B$ be a complete riemannian manifold with $C^{\infty}$ boundary $\partial B$ and $f a C^{\infty}$-function on $B$. Suppose that $B$ and $f$ satisfy the following conditions;

(1.B.1) $B$ has the riemannian simple double $2 B$,

(1.F.1) $f(x)>0$ if $x \in B \backslash \partial B$, and $f$ is an odd function on a neighbourhood of $\partial B$ of the arc-length $r$ in the inner normal direction to $\partial B$.

(1.F.2) $\|\operatorname{grad} f\|(x)=1$ if $x \in \partial B$.

Let $M$ be $(B \backslash \partial B) \times_{\left.f\right|_{B \backslash \partial B}} S^{n}(1)$. Then there is the unique complete riemannian manifold $\mathscr{M}$ without boundary such that $\mathscr{M}$ is the completion of $M$.

Proof. Let $(U, \varphi)$ be a local path of $\partial B$ and $N$ the $\varepsilon$-collar neighborhood of $U$ in $B$, We define a manifold $\mathscr{N}$ by

$$
\mathscr{N}:=(N \backslash U) \times_{f|N| U} S^{n}(1) .
$$

Imbedding of $S^{n}(1)$ into $R^{1+n}$, we define a diffeomorphism $\Psi$ of $\mathscr{N}$ into $R^{m} \times R^{1+n}$ by

$$
\Psi:((x, \exp r X), y) \longrightarrow(\varphi(x), r(y)),
$$

where $X \in T_{x} B$ is the unit inner normal vector to $\partial B$ and $0<r<\varepsilon$. 
We take the riemannian metric $g$ of $\Psi(\mathscr{N})$ so that $\Psi$ may become an isometry. Note that $g$ can be extended to the continuous metric $\bar{g}$ of $\overline{\Psi(\mathscr{N})}$ by the natural way. We have only to show that $\bar{g}$ is of class $C^{\infty}$ at the origin. Let $\left(x^{1}, \cdots, x^{m}, x^{m+1}, \cdots, x^{m+1+n}\right)$ be the Cartesian coordinates of $R^{m} \times R^{1+n}$. And we adopt the ranges of indices;

$$
1 \leq i, j \leq m \text { and } m+1 \leq \alpha, \beta \leq m+1+n .
$$

It is clear from Lemma 1.2 that $\bar{g}_{i j}:=\bar{g}\left(\partial / \partial x^{i}, \partial / \partial x^{j}\right)$ is of class $C^{\infty}$. It follows from Lemma 1.2 again that $(1 / r) \bar{g}\left(\partial / \partial x^{i}, \partial / \partial r\right)$ is of class $C^{\infty}$. Therefore $\bar{g}_{i \alpha}:=\bar{g}\left(\partial / \partial x^{i}, \partial / \partial x^{\alpha}\right)=x^{\alpha}(1 / r) \bar{g}\left(\partial / \partial x^{i}, \partial / \partial r\right)$ is of class $C^{\infty}$. Finally, we have that

$$
\begin{aligned}
\bar{g}_{\alpha \beta}: & =\bar{g}\left(\partial / \partial x^{\alpha}, \partial / \partial x^{\beta}\right) \\
& =\tilde{g}_{\alpha \beta}+\frac{f^{2}(x, r)-r^{2}}{r^{4}} r^{4} g_{S^{n}}\left(\partial / \partial x^{\alpha}, \partial / \partial x^{\beta}\right) \\
& =\tilde{g}_{\alpha \beta}+\frac{f^{2}(x, r)-r^{2}}{r^{4}}\left(r^{2} \tilde{g}_{\alpha \beta}-x^{\alpha} x^{\beta}\right)
\end{aligned}
$$

where $\tilde{g}$ is the standard metric on $R^{m} \times R^{1+n}$. It follows from Lemma 1.2 that $\left(f^{2}-r^{2}\right) / r^{4}$ is of class $C^{\infty}$. Therefore, $\bar{g}_{\alpha \beta}$ is of class $C^{\infty}$.

Remark 1.4 ([B] p. 269). If $m=0$ in Proposition 1.3, we can get a theorem of J. Kazdan-F. Warner; If we identify $\left\{x \in R^{1+n}|0<| x \mid<\varepsilon\right\}$ with $(0, \varepsilon) \times S^{n}$ in polar coordinates, the $C^{\infty}$-riemannian metric $d t^{2}+\varphi(t)^{2} \hat{g}_{0}$ (where $t$ is the parameter on $(0, \varepsilon)$ and $\hat{g}_{o}$ a metric on $S^{n}$ ) extends to a $C^{\infty}$-riemannian metric on $\left\{x \in R^{n}|| x \mid<\varepsilon\right\}$ if and only if $\hat{g}_{o}$ is $\lambda g_{\text {can }}$ where $g_{\text {can }}$ is the canonical metric on $S^{n}$ and $\lambda$ some positive constant, and $(1 / \lambda) \varphi$ is the restriction on $(0, \varepsilon)$ of $a C^{\infty}$ odd function on $(0, \varepsilon)$ with $(1 / \lambda) \varphi^{\prime}(0)=1$.

ObSERVATION 1.5. Since $\mathscr{M}$ is a completion of $M$ as a metric space, by means of theory of metric spaces, we can see that the condition (1.B.1) is necessary for the existence of $\mathscr{M}$. The condition (1.B.1) is strictly stronger than the condition that $\partial B$ is totally geodesic. For example, consider the surface of revolution of the graph

$$
x \in[0, \infty) \longrightarrow x^{3}-3 x^{2}+6 \in R .
$$

2.

Lemma 2.1 ([B-O]). Let $M:=B \times{ }_{f} F$ be a warped product with $a$ warping function $f$ where $B$ and $F$ are any riemannian manifolds. Let $\pi_{1}$ 
and $\pi_{2}$ be the natural projections of $M$ onto $B$ and $F$ respectively. Let $\Pi$ be a 2-plane tangent to $M$ at $x$ and $\{X+V, Y+W\}$ an orthonormal basis for $I I$, where $X, Y \in T_{\pi_{1}(x)} B$ and $V, W \in T_{\pi_{2}(x)} F$. The sectional curvature $K(\Pi)$ of $\Pi$ in $M$ is given by

$$
K(\Pi)=K_{X, Y}^{1}+K_{X, Y, W}^{2}+K_{V, W}^{3},
$$

where

$$
\begin{aligned}
& K_{X, Y}^{1}:=K_{B}(X, Y)\|X \wedge Y\|_{B}^{2}, \\
& K_{X, Y, V, W}^{2}:=-f\left(\pi_{1}(x)\right)\left\{\|W\|_{F}^{2}\left(\left(\nabla_{B}\right)^{2} f\right)(X, X)-2\langle V, W\rangle_{F}\left(\left(\nabla_{B}\right)^{2} f\right)(X, Y)\right. \\
& \left.\quad+\|V\|_{F}^{2}\left(\left(\nabla_{B}\right)^{2} f\right)(Y, Y)\right\}, \\
& K_{V, W}^{3}:=f^{2}\left(\pi_{1}(x)\right)\left\{K_{F}(V, W)-\|\operatorname{grad} f\|_{B}^{2}\right\}\|V \wedge W\|_{F}^{2},
\end{aligned}
$$

and $\nabla_{(.)}$and $K_{(.)}$are the covariant derivative and the sectional curvature of $($.$) respectively and \left(\nabla_{B}\right)^{2} f$ is the Hessian of $f$.

We shall prove Theorem. By the conditions of $B$, there is a diffeomorphism $\Psi: \partial B \times[0, \infty) \rightarrow B$ such that, for any $x \in \partial B, \tau_{x}(r):=\Psi(x, r)$ is the geodesic parametrized by the arc-length $r$, starting at $x$ and normal to $\partial B$. (Thus, Remark after Theorem holds.) Moreover, we have that $\pi_{1}(\mathscr{M})=\pi_{1}\left(\partial B \times R^{1+n}\right)=\pi_{1}(\partial B)=0$, because $\partial B$ is simply-connected by the conditions. Since Lemma 2.1, (B.2) and (F.2) imply that $K^{1}, K^{2}$ and $K^{3}$ are non-positive on $M$ and at least one of them is strictly negative on $M$, it is enough to show that at least one of $K^{1}, K^{2}$ and $K^{3}$ is strictly negative if $r \rightarrow 0$. Let $x_{o}$ be any point of $\partial B$ and $X_{r}, Y_{r}, V_{r}, W_{r}$ any vector fields along $\tau_{x_{0}}(r)$, where $X_{r}, Y_{r}$ are horizontal and $V_{r}, W_{r}$ are vertical if $r \neq 0$.

Case 1. The case that $X_{o}$ and $Y_{o}$ are linearly independent. We have

$$
K_{X_{0}, Y_{o}}^{1}<0 \text {. }
$$

Case 2. The case that $V_{o}$ and $W_{o}$ are linearly independent. (F.1) and (F.2) imply that

$$
f^{2}(r)=r^{2}+2 a r^{4}+\cdots, \quad a>0
$$

and

$$
\begin{aligned}
\|\operatorname{grad} f(r)\|_{B}^{2} & \geq\langle\operatorname{grad} f(r), \partial / \partial r\rangle_{B}^{2} \\
& =\left(\frac{\partial f}{\partial r}\right)^{2} \\
& =1+6 a r^{2}+\cdots .
\end{aligned}
$$


Then we have

$$
\begin{aligned}
\frac{1-\|\operatorname{grad} f(r)\|_{B}^{2}}{f^{2}(r)} & \leq \frac{1-\left(1+6 a r^{2}+\cdots\right)}{r^{2}+2 a r^{4}+\cdots} \\
& =\frac{-6 a+O(r)}{1+O(r)} .
\end{aligned}
$$

Therefore we have

$$
\lim _{r \rightarrow 0} K_{V_{r}, W_{r}}^{3} \leq-6 a<0 .
$$

Case 3. The case except Case 1 and Case 2. We can choose $X_{r}, Y_{r}$, $V_{r}$ and $W_{r}$ such that $Y_{r}=c_{1} X_{r}$ and $W_{r}=c_{2} V_{r}$, where $c_{1}$ and $c_{2}$ are constants with $c_{1} \neq c_{2}$. Let $\Pi_{r}$ be the 2-plane spanned by the orthonormal basis $\left\{X_{r}+V_{r}, Y_{r}+W_{r}\right\}$. Then we have

$$
K\left(\Pi_{r}\right)=-\frac{\left(\left(\nabla_{B}\right)^{2} f\right)_{X_{r}, X_{r}}}{f(r)\left\langle X_{r}, X_{r}\right\rangle_{B}}
$$

To get $\lim _{r \rightarrow 0} K\left(\Pi_{r}\right)<0$, it is enough to show that

$$
\lim _{r \rightarrow 0} \frac{\left(\left(\nabla_{B}\right)^{2} f\right)_{X_{r} \cdot X_{r}}}{f(r)}>0
$$

under the assumption $\left\|X_{r}\right\|_{B}=1$.

$$
\frac{\left(\left(\nabla_{B}\right)^{2} f\right)_{X_{r}, X_{r}}}{f(r)}=\frac{f^{\prime \prime}(r)\left(\nabla_{X_{r}} r\right)^{2}+f^{\prime}(r)\left(\nabla^{2} r\right)_{X_{r}, X_{r}}}{f(r)},
$$

and (F.2) imply the claim. Therefore we have Theorem.

EXAmple 2.2 (cf. [M]). Let $R^{m}$ be given a negatively curved metric, and $B:=[0, \infty) \times{ }_{\varphi} R^{m}$ the warped product with the warping function $\varphi$ such that (1) $\varphi$ is a $C^{\infty}$-even function in a neighbourhood of 0 , (2) $\varphi>0$, and (3) $\varphi^{\prime \prime}>0$. Then $B$ satisfies the conditions of Theorem.

Comment of counter example of M.T. Anderson. If, in Theorem, we set the following, we can get his example; $2 B:=H^{2 p}\left(-a^{2}\right), \partial B:=$ the totally geodesic hyperplane $H^{2 p-1}$ of $H^{2 p}\left(-a^{2}\right)$ and $f(r):=\sinh r$.

Acknowledgment. The authors would like to thank Professors J. Dodziuk and M. T. Anderson for their useful private letters. 


\section{REFERENCES}

[A] M. F. Atiyah, Elliptic operators, discrete groups and von Neumann algebras, Astérisque, 32-33 (1976), 43-72.

[An] M. T. Anderson, $L^{2}$-harmonic forms and a conjecture of Dodziuk-Singer, Bull. Amer. Math. Soc., 13 (1985), 163-165.

[B] A. L. Besse, Einstein manifolds, Ergeb. Math. Grenzgeb. 3. folge. Band 10, 1987, Springer-Verlag.

[B-O] R. L. Bishop-B. O'Neill, Manifolds of negative curvature, Trans. Amer. Math. Soc., 145 (1969), 1-49.

[D1] J. Dodziuk, $L^{2}$-harmonic forms on rotationally symmetric Riemannian manifolds, Proc. of Amer. Math. Soc., 77 (1979), 395-400.

[D2] J. Dodzuik, A private letter.

[K-W] J. L. Kazdan-F. W. Warner, Curvature functions for open 2-manifolds, Ann. of Math., 99 (1974), 203-219.

[M] H. Mori, Remarks on the Riemannian metric on the double of a manifold with boundary, preprint.

[O-N] T. Ochiai-J. Noguchi, Geometric Function Theory (in Japanese), Iwanami, 1986.

H. Kitahara

H. Kawakami

Department of Mathematics

Kanazawa University

Kanazawa, 920 Japan

Jin Suk Pak

Department of Mathematics

Kyungpook National University

Taegu, 635 Korea 